\title{
Numerical methods for quantitative finance
}

\author{
JAMIE ALCOCK
}

In this thesis the problems associated with Monte-Carlo pricing and hedging of financial claims contingent upon a supervolatile underlying security are considered. The motivation for these considerations is to price options for the Queensland electricity spot market.

The presence of supervolatility, which can be represented using a diffusion process but is often represented in the form of a jump-diffusion process or Lévy process, presents many challenges for any numerical pricing method including Monte-Carlo pricing. Some of these difficulties have been addressed in this thesis to price fairly, using Monte-Carlo pricing, a series of Asian style options on the Queensland electricity spot price.

In recent years the Australian National Electricity Market (the NEM) has been undergoing the transition to a fully deregulated marketplace. In December 1998, for the first time, the wholesale price of electricity was subject to market forces. The NEM includes the Queensland, New South Wales, Victorian, South Australian and Australian Capital Territory electricity markets, with Tasmania set to join in 2005. Almost 8 million end users are supplied by the world's longest interconnected power system, and the NEM trades up to $\$ 7$ billion of electricity annually ([6]).

The National Electricity Market Management Company Limited (NEMMCO) was established in 1996 to manage the NEM, a role which carries with it the responsibility for setting the spot price. The spot price is determined via a sellers Dutch auction. Each day, each generator submits a complex bid of prices and volumes. The demand fluctuates throughout the day, and every 5 minutes short-term supply and demand are realigned by NEMMCO. The 5 minute dispatch price for all bidders is set to the winning bid of the marginal supplier, and six sequential dispatch prices are averaged to determine the half-hour spot price. All successfully bidding generators receive the spot price for their product.

The introduction of market forces to the NEM has provided a plethora of challenges for mathematicians $([1,7,3])$ and economists $([11,10,8])$, amongst others. One of the

Received 16th May, 2005

Thesis submitted to The University of Queensland, September 2004. Degree approved, April 2005. Supervisor: Professor Kevin Burrage.

Copyright Clearance Centre, Inc. Serial-fee code: 0004-9727/05 \$A2.00+0.00. 
more pressing challenges, from the view of the financial manager of a market participant, involves the provision of risk management products.

For example, consider the position of an electricity generator. In order for a generator to be profitable, the average wholesale selling price of electricity must be higher than their average total cost of production (which will include significant fixed costs). However, the spot price of electricity fluctuates quite dramatically at times, so an open exposure to such fluctuating prices can inflict heavy losses (and at other times, handsome profits) upon a generating company. Unlike other commodities, electricity cannot be stored so an electricity generator cannot simply refuse to sell if the market price is too low. The electricity that is produced must be consumed instantly for the generator and NEM to remain stable. Risk management products such as options contracts, written on the spot price of electricity, are required to reduce the exposure of electricity generators to the fluctuations of the spot market.

Any reduction in market risk exposure faced by a market participant will ultimately result in reduced losses and increase profits. Consequently risk management products have real economic value. The challenge for financial mathematicians is to determine the fair price and efficient hedge of relevant financial instruments.

The problem of pricing and hedging risk management products for other commodities and financial securities (such as shares in a public company) was first solved in a modern way by the Nobel prize winning Black, Scholes and Merton ([2, 4]). However modern pricing methodologies rely heavily upon many restrictive assumptions on the underlying commodity/security. As is explored in Chapter 1 of the thesis, the electricity spot market violates these assumptions sufficiently to render previous models and analysis of risk management products inapplicable with respect to electricity markets. The electricity spot price process exhibits supervolatile properties, and this characteristic is the primary source of option pricing and hedging difficulties.

Thus a method of pricing and hedging electricity options is required so that market participants can confidently manage their exposure to market risk. While this problem has motivated the work in this thesis, the solutions found are more widely applicable to general contingent claim analysis than to the electricity markets alone. Any process that exhibits supervolatile properties will provide a similar mathematical challenge and so the results found in this thesis can be used to construct and price options on any supervolatile process.

Stochastic Ordinary Differential Equations (SODEs) are used to model the various diffusion processes throughout this dissertation. While the pathwise properties are not always required for pricing contingent claims, information about the path is required for determination of efficient hedges for the claims. Thus the numerical pricing and hedging schemes required here will necessitate the efficient and stable solution to SODEs that model supervolatile stochastic processes. Early in the thesis (Chapter 2) these back- 
ground issues are introduced and explored. The stability properties of many numerical methods make these schemes inappropriate for solving SODEs that model supervolatile processes. Furthermore the inadequacy of the most commonly utilised stochastic model, geometric Brownian motion (GBM), for modelling supervolatile process is discussed.

Indeed numerous new technologies were developed in order to numerically price and hedge options on a supervolatile underlying, including:

- Parametric estimation for various models of supervolatile processes (Chapter 3)

- Stable numerical schemes for solving SODEs that model supervolatile processes (Chapters 4, 5)

- A risk-neutral Monte-Carlo pricing scheme (Chapter 6)

The emphasis throughout this thesis was to develop generic, model independent methods that may be applied to a broad range of supervolatile models.

Parameter estimation for geometric Brownian motion has been well studied, but estimating parameters of Lévy SODEs, jump-diffusions or other models of supervolatile processes is still an unresolved problem. In Chapter 3, a simulation based method that utilises genetic algorithms to estimate parameters of this class of SODEs (GePERs) is presented and examined. This general method provides consistent estimates for the parameters of any Markovian SODE. In this setting, where we examine several different SODEs using the same data set, such a general estimation method proves particularly suitable.

One class of numerical schemes, called the Balanced method [5], has been introduced recently to solve stiff systems of SODEs, including supervolatile systems. In Chapter 4 the stability properties of this method are examined and compared to those of a traditional method of the same order of convergence. The anticipated asymptotic and mean-square stability benefits of the Balanced method relative to the explicit Euler-Maruyama scheme are formally exposed here. These stability properties are demonstrated by solving the stochastic Duffing-Van der Pol equation.

The techniques employed by this class of schemes provide inspiration for the development of another class of numerical methods (SSO1) to solve stiff SODEs, which are described in Chapter 5. Strong order convergence is demonstrated for this class of schemes, as well as a proof of strong order convergence for similar schemes based upon the same numerical principles. Stability analysis suggests that this class of schemes is particularly well suited for solving stiff SODEs using large stepsizes. This class of schemes are used to solve Sagirow's satellite problem [9].

The identification of risk-neutral measures for the models described in Chapter 2 is not clear. A recently developed numerical method of doing this for historical data [12], using the minimum entropy change of measure, is examined in Chapter 7. Also a nonparametric method of hedging and forming a replicating portfolio is developed, and 
the error-metrics of this hedge, along with the error metric of the nonparametric pricing method are examined. Finally the maximum-entropic principles are used to develop a method of risk-neutral Monte-Carlo pricing that can be applied to a large class of stochastic models.

All these developments are amalgamated in the Chapter 7 in order to price an option on a supervolatile security. The motivating example of an Asian style option on the electricity spot price is priced using the methods developed in Chapters 3-6.

\section{REFERENCES}

[1] C. Baloi, J. Belward, and M. Bulmer, 'Short-term forecast of the load demand in Victoria', in International Congress on Modelling and Simulation (Modelling and Simulation Society of Australia and New Zealand Inc., Canberra, 2004), pp. 1065-1070.

[2] F. Black and M. Scholes, 'The pricing of options and corporate liabilities', J. Political Economy 81 (1973), 637-654.

[3] L.Clewlow and C. Strickland, Energy derivatives: Pricing and risk management (Lacima Publications, Sydney, London 2000).

[4] R. Merton, 'Theory of rational option pricing', Bell J. Econ. and Management Sci. 4 (1973), 141-183.

[5] G. Milstein, E. Platen, and H. Schurz, 'Balanced implicit methods for stiff stochastic systems', SIAM J. Num. Anal. 35 (1998), 1010-1019.

[6] NEMMCO Ltd, Melbourne, An Introduction to Australia's national electricity market, 2004.

[7] D. Pilipovic, Energy risk: Valuing and managing energy derivatives (McGraw-Hill, New York, 1998).

[8] G. Rothwell and T.Gómez, Electricity economics: Regulation and deregulation (Wiley, Hoboken, 2003).

[9] P. Sagirow, Stochastic methods in the dynamics of satellites, CISM Lecture Notes $\mathbf{5 7}$ (Udine, 1970).

[10] F. Schweppe, M. Caramanis, R. Tabors, and R. Bohn, Spot pricing of electricity (Kluwer Academic Publishers, Boston, 1988).

[11] P. Simshauser, Microeconomic reform of wholesale power markets: $A$ dynamic partial equilibrium analysis of negative externalities, (Ph.D. thesis) (The University of Queensland, Australia, 2001).

[12] M. Stutzer, 'A simple nonparametric approach to derivative security valuation', J. Finance 51 (1996), 1633-1652.

UQ Business School

University of Queensland

Brisbane 4072, Australia

e-mail: j.alcock@business.uq.edu.au 